\title{
Scanning two memorized lists
}

\author{
GAIL ELKIND and MICHAEL C. CORBALLIS \\ University of Auckland, Auckland, New Zealand
}

\begin{abstract}
Subjects were timed as they decided whether singly presented probe words belonged to one or the other of two memorized lists, or to neither list. Each list varied in length from one to four words. Reaction times increased linearly with the combined number of words in the two lists. When there was no a priori basis for distinguishing the lists, the slope of the function for positive test probes was 33-35 msec per word higher than that for negative probes. The slope for negative probes was $58 \mathrm{msec}$ per word in one experiment and $46 \mathrm{msec}$ per word in another. This suggests that subjects first scanned the combined lists exhaustively to determine whether the probe was present; if it was not, they made a negative response, and if it was, they scanned again to determine which list it was in. When the words in the two lists were conceptually distinct (one list representing animate and the other inanimate objects), the difference in slope was reduced to only 6 msec per word, suggesting that the second scan was all but eliminated.
\end{abstract}

In the item-recognition task pioneered by Sternberg $(1966,1969 a, 1969 b, 1975)$, the subject memorizes a short list of items and is then presented with a probe item. Sternberg found that reaction time (RT) to decide whether or not the probe was a member of the memorized list was a linear function of the number of items in the list, a finding that has proven remarkably robust, despite variations in the type of stimuli used and in procedure (for reviews, see Corballis, 1975; Sternberg, 1975). To explain this finding, Sternberg suggested that the decision was achieved by a serial exhaustive scan of the list. The exhaustive nature of the scan was inferred from the fact that the slope of the function relating RT to list length was the same for positive and negative probes. Although other authors have proposed alternative models, based, for instance, on direct access to the representation of the probe (e.g., Corballis, 1967; Corballis, Kirby, \& Miller, 1972; Norman \& Wickelgren, 1969) or on parallel search (e.g., Ratcliff, 1978), the notion of serial exhaustive scanning has persisted; for example, it is currently at the center of a controversy concerning the nature of the distinction between controlled and automatic processing (Ryan, 1983; Shiffrin \& Schneider, 1984).

It has been suggested that under some circumstances, subjects may be able to simultaneously carry out two separate scans of memorized information, apparently without mutual interference. For instance, there is evidence that subjects may scan a list simultaneously for formal and semantic information (Burrows \& Okada, 1973) and simultaneously for physical and categorical codes (Burrows \& Okada, 1974). In another experiment, Burrows and Solomon (1975) presented two lists of letters simultaneously, one visually and the other auditorily, and showed that scanning for a single probe from either list was at least as fast as when visual and auditory lists were

Reprint requests should be mailed to M. C. Corballis, Department of Psychology, University of Auckland, Auckland, New Zealand. presented alone. They interpreted this to mean that the subjects scanned the visual and auditory lists simultaneously. In an earlier experiment, Burrows (1972) found no evidence that subjects scanned visual and auditory lists simultaneously when the items had been presented one at a time, with visual and auditory items randomly intermixed.

Ryan (1983) recently claimed further evidence for the simultaneous, independent scanning of two lists, a fixed list presented prior to a sequence of test trials and a varied list that varied from trial to trial. The subject's task was simply to decide whether each of a series of test probes was in the fixed or the varied list. According to Ryan, the RT data from an experiment of his own, as well as data from one reported by Forrin and Morin (1969), imply that subjects scan serially but concurrently through each of the fixed and varied lists, and that the rate of concurrent scanning is equal to that of scanning a varied list alone. However, this conclusion is at best premature, since Ryan's analysis is based on the mistaken premise that successive scanning of the two lists should increase the slope of the function relating RT to list length; in fact, the data appear to be as compatible with successive as with simultaneous scanning of the two lists (Corballis, 1986).

One factor that might influence whether separate lists are scanned simultaneously or successively is the response requirement. This possibility is suggested by research on the detection of targets among signals that are presented in simultaneous pairs (e.g., Ostry, Moray, \& Marks, 1976; Sorkin, Pohlmann, \& Woods, 1976). In a review of this work, Duncan (1980) concluded that if subjects are required to make a single response to a target, regardless of which stream of signals it appears in, then any effects of divided attention are at best slight. However, if the subjects are required to make separate responses depending upon which stream the target appears in, such as pressing a left-hand button if a target is in the left ear and a right-hand button if the target is in the right ear (e.g., 
Ostry et al., 1976), then there are substantial effects of divided attention. That is, in the latter case, performance in responding to targets in one stream is lowered if there is a simultaneous target in the other.

This raises the question of whether a similar rule might operate in the detection of probes in memorized lists (as distinct from the detection of targets in lists presented "online'). Thus, in Burrows and Solomon's (1975) experiment, for instance, subjects were required to make only a single positive response to any given probe, regardless of which of the two simultaneously presented lists it appeared in. That they were apparently able to scan the lists simultaneously suggests little influence of divided attention. However, if subjects are required to make separate "positive" responses, depending upon which of two lists a probe is in, then one might expect attention to be directed to only one list at a time, implying successive scanning.

In the present study, therefore, we explored a paradigm in which subjects were given two lists to memorize and were then presented with a series of probes; their task was to press one button if a probe was in one list, another button if it was in the other, and a third button if it was in neither. Thus, there were three response alternatives rather than only the two offered in previous experiments on memory scanning. We also included a control condition, in which the words in the two sets were incorporated into a single list, and the response choice was reduced to a dichotomous one. The conditions were similar to those of previous experiments (e.g., Corballis, Katz, \& Schwartz, 1980; Corballis \& Miller, 1973; Hockley \& Corballis, 1982) that have yielded linear set-size functions for single memorized lists, at least over the range of total memory loads (two to eight words) used in the present study.

Our main interest was whether the subjects would scan the two lists simultaneously, or one list after the other. For the present, we assume that scanning will be serial and exhaustive and defer until the General Discussion the question of whether the results might be interpreted in terms of processes other than serial scanning. We also assume that variations in RT with the lengths of the lists are due to the scanning components, and not to other components of the RT equation. We now consider the implications of simultaneous and successive scanning.

If we assume that the subjects scan the two lists simultaneously, we can further distinguish two possibilities. One is that a positive decision might be reached after the subject has scanned exhaustively through the list containing the probe, regardless of the length of the other list, whereas negative decisions would be reached after the subject has scanned exhaustively, but simultaneously, through both lists. This implies that positive RTs would be proportional to the list length associated with each response. If the time taken to scan each item is constant for each subject, negative RTs should be proportional to the length of the larger of the two lists. The second possibility is that subjects might scan both lists exhaustively, but simultaneously, before reaching either positive or negative de- cisions. Again assuming constant scan time, RT to both positive and negative probes should then be proportional to the length of the longer of the two lists in each condition.

These predictions are complicated somewhat if scan times are variable. With variable scan times, it will in general take longer to scan two equal lists than to scan a single list of the same length. Even if the lists are unequal, scanning may take longer than the time required to scan the longer list, since by chance long scan times may be allocated to the shorter list and short scan times to the longer list. In the absence of knowledge about the distribution of scan times, we cannot make exact predictions; in the Results section of Experiment 1, however, we describe predictions based on the assumption that scan times are uniformly distributed.

However, if we assume that the subjects scan successively through the two lists, whether scan time is constant or variable, we might then expect that RT would be a linear function of the combined number of words in the two lists.

\section{EXPERIMENT 1}

\section{Method}

Subjects. The subjects were 11 men and 9 women, aged between 18 and 32 years except for one 53-year-old man in the group that learned two lists. Since this older man was in the middle of the range for that group in both RT and accuracy, we felt that his inclusion did not distort the comparison between groups. Without this subject, the mean ages of the groups were 23.7 and 24.6 years for the groups who learned one and two lists, respectively.

Stimuli and Apparatus. The stimuli were words, randomly selected from a pool of 32 common, concrete, one-syllable nouns, each three to five letters long. All were chosen originally from Thorndike and Lorge's (1944) lists of A- or AA-frequency words, and were scored by Paivio, Yuille, and Madigan (1968) as being above 6.0 in imagery, above 6.7 in concreteness, and above 5.1 in meaningfulness.

The experiment was controlled by a PDP-11/03 computer with an attached Diablo 166A printer, which printed out the lists for the subjects to memorize, and a Tektronix 5403 table-mounted storage oscilloscope, which displayed the probe words. Each letter was $6.8 \mathrm{~mm}$ in height and $4.0 \mathrm{~mm}$ in maximum width, and was in uppercase. The screen was about $50 \mathrm{~cm}$ from the subject's eyes.

The response buttons were arranged at the corners of an isosceles triangle with sides of $35 \mathrm{~mm}, 35 \mathrm{~mm}$, and $50 \mathrm{~mm}$. A button labeled " $A$ " was on the left base, a button labeled " $B$ " on the right base, and button labeled " $C$ " on the vertex. The subjects waited between trials with the forefinger of the preferred hand at a point equidistant from the three buttons, and moved that finger to the appropriate button to register their decisions.

Procedure. The subjects were randomly allocated to two groups. Subjects in the "one-list" group, Group 1L, learned lists of two, three, four, six, and eight words. For each subject in this group, a matched subject in the "two-list" group, Group 2L, learned the same words divided into 2 lists, Lists A and B. For instance, if a Group $1 \mathrm{~L}$ subject learned a four-word list, the matched Group $2 \mathrm{~L}$ subject might learn the same four words as 2 two-word lists. Each subject worked through 10 lists or pairs of lists on each daily session. The list lengths for the 10 pairs of lists were as follows: $1 / 1$, $1 / 2,2 / 1,2 / 2,1 / 3,3 / 1,3 / 3,2 / 4,4 / 2$, and $4 / 4$, where the first number in each case refers to the length of List $A$ and the second to the length of List B. The different conditions were characterized by 
response inconsistency (Schneider \& Shiffrin, 1977). That is, selection was random for each subject pair and condition, so that a given word might appear in List A in some conditions, in List B in others, and in neither list in others. There was no repetition of words within conditions.

After learning a list or pair of lists, each subject was then presented with a series of 36 test probes, of which 24 were positive and 12 negative. For Group $2 \mathrm{~L}, 12$ of the positive probes came from List A and 12 from List B. Each positive probe appeared in each series an equal number of times, whereas negative probes were never repeated within the series for that condition. For example, if a particular list contained 2 words, each was presented six times in the series, and the 12 randomly chosen negative probes were presented only once each. Subjects in Group $2 \mathrm{~L}$ were instructed to press Button A if the probe was in List A, Button B if it was in List B, and Button $C$ otherwise. Subjects in Group 1L were told to press Button $\mathrm{B}$ (on the right) if the probe was in the memorized list and Button $\mathrm{C}$ otherwise. All subjects were told to respond as quickly as possible without making errors.

The subjects in Group $2 \mathrm{~L}$ were given both lists on a computer printout, headed "List A" and "List B." They were printed in columns with List A above List B. For subjects in Group 1L, the list was presented in a single column. The subjects were given as much time as they wanted to memorize the lists, but were required to recall the words correctly and in their correct lists before the probe series commenced. When ready, the subject initiated the series by pressing any of the three buttons. At $500 \mathrm{msec}$ after the button was released, the first probe appeared. Each probe remained on the screen until the subject responded, and the next probe appeared $500 \mathrm{msec}$ after the button was released. After the 36 probes had been responded to, the subject learned the list or lists for the next condition. The conditions were presented in a different random order for each matched pair of subjects.

Each subject was tested in four separate sessions, and in each session received all 10 conditions, in random order, making a total of 360 trials per session. These sessions were usually on separate days, and all occurred within 5 days. The first session was treated as practice and was excluded from the analysis of results.

\section{Results}

Reaction times. First, an overview: The main results were that RT in Group 2L depended on total memory load rather than on the length of the specific list containing the probe or on the allocation of load between the lists. However, the slope of the RT function for positive probes was nearly twice that for negative probes. In Group 1L, by contrast, the slope for positive probes was slightly less than that for negative probes. These features of the data are shown in Figure 1.

In the following analyses, mean RTs were computed for correct responses to each type of probe (A, B, or negative) under each condition of list length, for each subject.
The data were first analyzed for Group $2 \mathrm{~L}$ only, in order to determine whether there were systematic effects related to the comparison between List A and List B probes, as suggested by the hypothesis of simultaneous scanning mentioned in the introduction. According to one version of this hypothesis, described in the introduction, one would expect RT to List A probes to be linearly related to the length of List $A$, and RT to List $B$ probes to be linearly related to the length of List $\mathrm{B}$, in each case regardless of the length of the other list. This implies an interaction between list-length condition and positive probe type (A, B). However, this interaction was not significant $[F(9,162)=1.33, M S e=20,986]$, thus failing to support any relation between a positive response and the list length associated with it.

The mean RT to List A probes $(829 \mathrm{msec})$ was somewhat shorter than that to List B probes $(882 \mathrm{msec})$. This difference may be due to the physical layout of the buttons and does not seem relevant to hypotheses about scanning, since it was independent of condition; in any case, it was not significant $[t(18)=1.84, M S \mathrm{e}=123,349]$. None of the other effects associated with the comparison between A and B responses was significant either. The RTs to List A and List B probes were therefore averaged, and the probe factor reduced to the comparison of positive (A and B combined) with negative probes. This enabled us to incorporate both groups in the same overall analysis of variance; group was treated as a betweensubjects factor and condition and probe type as withinsubjects factors.

This overall analysis revealed a significant main effect of group $[F(1,18)=16.37, M S \mathrm{Se}=633,464, p<.001]$, and significant interactions between group and probe type $[F(1,18)=26.37, M S \mathrm{e}=83,287, p<.001]$, between group and condition $[F(9,162)=7.53, M S e=23,064$ $\mathrm{p}<.001]$, and between group, probe type, and condition $[F(9,162)=8.23, M S \mathrm{e}=7,965, p<.001]$. Mean RTs for each group, probe type, and condition are shown in Table 1.

In order to analyze the group interactions further, separate analyses of variance were carried out for each group. Separate analyses were preferred to tests of simple main effects and simple interactions because the error terms in the Group $2 \mathrm{~L}$ analysis were systematically higher than the corresponding error terms in the Group $1 \mathrm{~L}$ analysis, indicating heterogeneity of variance between

Table 1

Mean RTs for Correct Responses to Each Probe Type Under Each Condition for Each Group in Experiment 1

\begin{tabular}{clrlllllllllr}
\hline & & \multicolumn{10}{c}{ Condition* } \\
\cline { 2 - 12 } & Group & Probe & $1 / 1$ & $1 / 2$ & $2 / 1$ & $2 / 2$ & $1 / 3$ & $3 / 1$ & $3 / 3$ & $2 / 4$ & $4 / 2$ & $4 / 4$ \\
\hline \multirow{2}{*}{$1 \mathrm{~L}$} & A(Pos) & 499 & 530 & 545 & 560 & 564 & 557 & 635 & 617 & 633 & 662 \\
& C(Neg) & 627 & 651 & 666 & 690 & 682 & 690 & 806 & 802 & 781 & 829 \\
& A(Pos) & 596 & 638 & 741 & 779 & 706 & 787 & 1013 & 938 & 956 & 1138 \\
$2 \mathrm{~L}$ & B(Pos) & 637 & 756 & 731 & 846 & 822 & 809 & 1080 & 986 & 954 & 1196 \\
& C(Neg) & 664 & 719 & 709 & 752 & 834 & 802 & 946 & 907 & 917 & 977 \\
\hline
\end{tabular}

*Conditions labeled by assignment of list lengths to Lists A and B, respectively, in Group $2 \mathrm{~L}$. 
groups. This heterogeneity was significant in the case of the error terms for condition $[F(81,81)=3.40$, $p<.001]$ and for the condition $\times$ probe type interaction $[F(81,81)=1.82, p<.01]$.

For Group $1 \mathrm{~L}$, there was a significant effect of condition $[F(9,81)=22.82, M S e=10,493, p<.001]$, which was attributable almost entirely to the linear trend component of memory load $[F(1,81)=197.57, p<.001]$, leaving an insignificant residual $[F(8,81)=0.98]$. This linear trend accounted for $96.19 \%$ of the variance between conditions, and represented a slope, or scanning rate, of $34 \mathrm{msec}$ per word. Responses to positive probes were significantly faster than responses to negative probes $[F(1,9)=72.41, M S e=41,987, p<.001]$; the means were $580 \mathrm{msec}$ and $722 \mathrm{msec}$, respectively. This difference may have been due in part to the arrangement of the response buttons. Although the slope of the best-fitting linear function was somewhat less for positive $(28 \mathrm{msec}$ per word) than for negative ( $39 \mathrm{msec}$ per word) probes, the interaction between probe type and condition was not significant $[F(9,81)=1.51, M S \mathrm{e}=5,625]$. Figure 1 shows RT plotted against memory load for both positive and negative probes.

For Group $2 \mathrm{~L}$, the interaction between probe type and condition was highly significant $[F(9,81)=8.36, M S \mathrm{~S}$ $=10,279, p<.001]$. Tests of simple main effects showed a significant effect of condition for both positive $[F(9,124)$ $=37.48, M S \mathrm{e}=22,957, p<.001]$ and negative probes $[F(9,124)=15.86, M S e=22,957, p<.001]$; the error term and its degrees of freedom were calculated according to the formulas given by Winer (1971, pp. 544-545).

On the assumption that variations between conditions were due entirely to the scanning component, we next tested three scanning models as possible explanations of the effect of condition on RT: (1) The first model assumes that each subject scans both lists simultaneously and ex- haustively, with constant scan time per word. As shown in the first row of Table 2 , this model predicts that RT will be proportional to the larger of the two list lengths in each condition. (2) The second model also assumes simultaneous exhaustive scanning of the two lists, but scan time for each word is now assumed to be variable. We cannot test this model precisely, since we do not know the distribution of scan times. To gain some impression, however, we assumed a uniform distribution of scan times between 0 and 2 units, with a mean of 1 unit, and generated 1,000 pairs of scans through the list lengths under each condition using the random number generator of the Apple II+ computer. This produced the expected mean scan times, rounded to two decimal places, shown in the second row of Table 2 . We suspect that the variance of this distribution is probably greater than that of any actual distribution of scan times, and that the difference between the predictions of constant and variable scan time is, if anything, exaggerated. (3) The third model assumes sequential exhaustive scanning of the two lists, so that RT is proportional to the sum of the lengths of the lists. This is shown in the third row of Table 2.

For positive responses, these models accounted for $68.2 \%, 87.1 \%$, and $97.1 \%$, respectively, of the variance between conditions. The first two left significant residuals $[F(8,124)=13.41$ and 5.56, respectively, $p<.01]$, whereas the third did not $[F(8,124)=1.28]$. For negative responses, the models explained $83.7 \%, 94.2 \%$, and $92.7 \%$, respectively; the first left a significant residual $[F(8,124)=2.90, p<.01]$, but the second and third did not $[F(8,124)=1.03$ and 1.30 , respectively $]$. Although we cannot entirely rule out simultaneous scanning with variable scan times, therefore, the results are more generally supportive of sequential scanning of the two lists.

We must still account for the interaction between probe type and condition, however; according to the sequential-

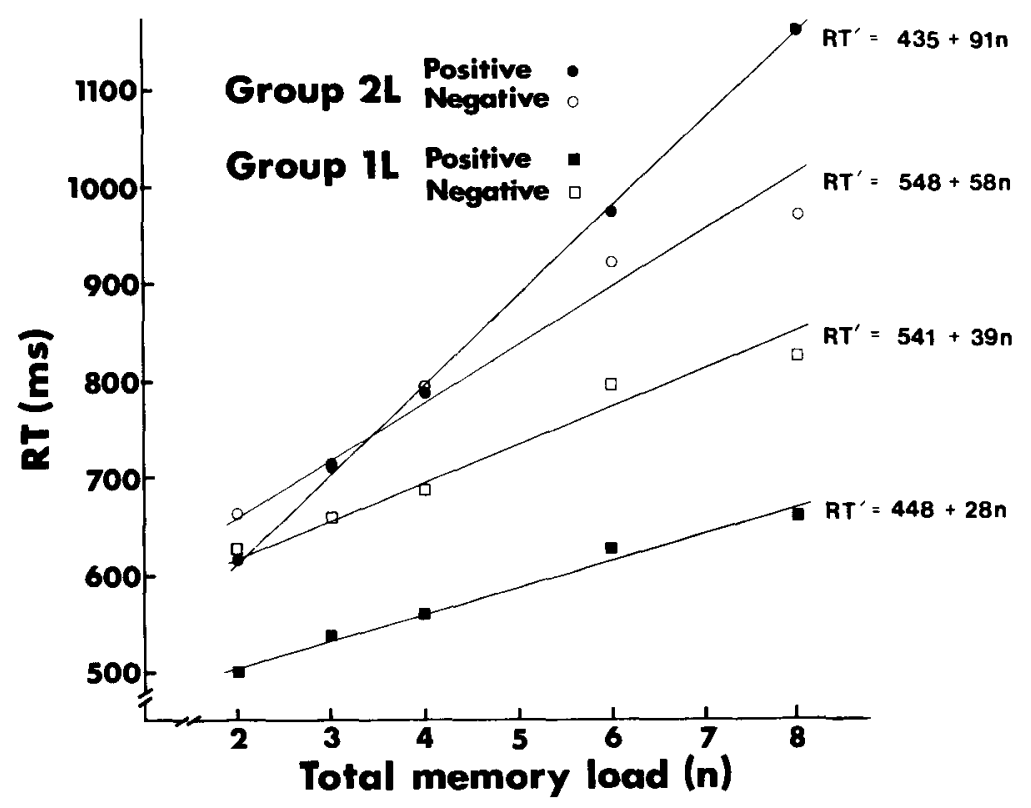

Figure 1. Mean RT to positive and negative probes plotted against memory load, for each group in Experiment 1. 
Table 2

Predicted Scan Times, Based on Unit Mean Scan Time per Word, Associated with Each Condition, According to Three Different Scanning Models, for Group 2L in Experiment 1 and Group $M$ in Experiment 2

\begin{tabular}{|c|c|c|c|c|c|c|c|c|c|c|}
\hline \multirow[b]{2}{*}{ Model } & \multicolumn{10}{|c|}{ Condition } \\
\hline & $1 / 1$ & $1 / 2$ & $2 / 1$ & $2 / 2$ & $1 / 3$ & $3 / 1$ & $3 / 3$ & $2 / 4$ & $4 / 2$ & $4 / 4$ \\
\hline Simultaneous* & 1 & 2 & 2 & 2 & 3 & 3 & 3 & 4 & 4 & 4 \\
\hline Simultaneous $\dagger$ & 1.36 & 2.11 & 2.11 & 2.44 & 3.02 & 3.02 & 3.59 & 4.04 & 4.04 & 4.69 \\
\hline Sequential & 2 & 3 & 3 & 4 & 4 & 4 & 6 & 6 & 6 & 8 \\
\hline
\end{tabular}

*Based on constant scan time per comparison. †Based on variable scan time per comparison.

Table 3

Percentage of Correct Responses to Positive and Negative Probes Under Each Condition for Both Groups in Experiment 1

\begin{tabular}{|c|c|c|c|c|c|c|c|c|c|c|c|}
\hline \multirow[b]{2}{*}{ Group } & \multirow[b]{2}{*}{ Probe } & \multicolumn{10}{|c|}{ Condition } \\
\hline & & $1 / 1$ & $1 / 2$ & $2 / 1$ & $2 / 2$ & $1 / 3$ & $3 / 1$ & $3 / 3$ & $2 / 4$ & $4 / 2$ & $4 / 4$ \\
\hline $1 \mathrm{~L}$ & $\begin{array}{l}\text { Pos } \\
\text { Neg }\end{array}$ & $\begin{array}{l}99.31 \\
97.50\end{array}$ & $\begin{array}{l}99.17 \\
97.78\end{array}$ & $\begin{array}{l}97.50 \\
97.22\end{array}$ & $\begin{array}{l}97.92 \\
96.94\end{array}$ & $\begin{array}{l}99.17 \\
97.50\end{array}$ & $\begin{array}{l}98.19 \\
97.78\end{array}$ & $\begin{array}{l}97.78 \\
95.28\end{array}$ & $\begin{array}{l}96.67 \\
94.72\end{array}$ & $\begin{array}{l}97.08 \\
96.39\end{array}$ & $\begin{array}{l}93.33 \\
94.44\end{array}$ \\
\hline $2 \mathrm{~L}$ & $\begin{array}{l}\text { Pos } \\
\text { Neg }\end{array}$ & $\begin{array}{l}97.78 \\
98.33\end{array}$ & $\begin{array}{l}97.92 \\
99.44\end{array}$ & $\begin{array}{l}97.64 \\
99.17\end{array}$ & $\begin{array}{l}94.44 \\
98.06\end{array}$ & $\begin{array}{l}96.25 \\
99.44\end{array}$ & $\begin{array}{l}94.03 \\
96.39\end{array}$ & $\begin{array}{l}79.17 \\
96.39\end{array}$ & $\begin{array}{l}85.83 \\
96.39\end{array}$ & $\begin{array}{l}84.44 \\
95.83\end{array}$ & $\begin{array}{l}77.22 \\
95.56\end{array}$ \\
\hline
\end{tabular}

scanning model, the estimated scanning rate was $91 \mathrm{msec}$ per word for positive probes and $58 \mathrm{msec}$ per word for negative ones; the difference of $33 \mathrm{msec}$ per word was highly significant $[F(1,81)=48.34, M S \mathrm{e}=10,279$, $p<.001]$. One possible explanation is that the subjects scanned the lists twice in making positive decisions, but only once in making negative ones, with the extra positive scan taking $33 \mathrm{msec}$ per word, a rate that is well within the range of estimates normally obtained in memory-scanning experiments. However, the slope for negative RTs was higher in Group 2L (58 msec per word) than in Group 1L ( $39 \mathrm{msec}$ per word); this difference was tested by computing the simple interaction (Winer, 1971) between groups and the linear component of total memory load, which proved significant $[F(1,262)=10.25, M S e$ $=15,515, p<.001]$. The estimated rate of the first scan was thus somewhat higher than expected from the scanning of single lists.

Accuracy. The number of correct responses to each type of probe was counted for each subject under each condition. Analyses of variance of these scores showed essentially the same pattern of results as for RT. For Group 1L, the only significant effect was that of condition $[F(9,81)=5.46, M S \mathrm{e}=0.3305, p<.001]$. For Group $2 \mathrm{~L}$, there were significant effects due to probe type (A, B, negative) $[F(2,18)=17.57, M S \mathrm{e}=4.3886$, $p<.001]$ and to the interaction between probe type and condition $[F(18,162)=4.00, M S \mathrm{e}=1.8043, p<.001]$. Neither effect proved significant when the effect of probe type was reduced to the comparison between List $A$ and List $\mathrm{B}$ probes (both $F \mathrm{~s}<1$ ). This lack of interaction again suggests that relative responding to $A$ and $B$ probes did not depend on the relative assignment of the number of words to each list. Correct responses to List $\mathrm{A}$ and List B probes were therefore averaged for comparison with responses to negative probes. Table 3 shows the mean percent correct for positive and negative probes for each condition, for each group.

Finally, a combined analysis across groups revealed a significant main effect of group $[F(1,18)=8.61, M S \mathrm{e}=$ $4.4309, p<.01]$, significant interactions of group with probe type $[F(1,18)=22.49, M S e=3.3354, p<.001]$ and a significant triple interaction between group, probe type, and condition $[F(9,162)=5.22, M S e=0.8806$, $p<.001]$. The main contributor to these effects is the deterioration in accuracy of responses to positive probes with increasing memory load shown by Group $2 \mathrm{~L}$.

These results mirror the RT trends in that increasing RT is generally associated with decreasing accuracy. The RT trends cannot therefore be attributed simply to speed-accuracy trade-off.

\section{Discussion}

These analyses, and the plots shown in Figure 1, reveal that the functions were predominantly linear, consistent with serial scanning, for both groups. Moreover, the data suggested that the subjects in Group $2 \mathrm{~L}$ scanned the two lists successively rather than simultaneously. However, the slopes were considerably steeper in Group 2L than in Group 1L. In Group 2L, moreover, the slope for positive responses was significantly steeper than that for negative responses, whereas in Group $1 \mathrm{~L}$, it was the slope for negative responses that was the steeper.

To explain the result for Group $2 \mathrm{~L}$, we suggested that the subjects may have scanned the lists twice before making positive responses. The first scan simply established whether the probe was positive or negative. If it was negative, the subject then pressed Button C; but if it was positive, he or she scanned the lists again in order to establish which list it was in, and then pressed Button $A$ or $B$ accordingly. The slope of the negative function provides an estimate of the rate of the first scan, which is 
about 58 msec per word, while the difference in slope between positive and negative functions provides an estimate of the rate of the second scan, which is about 33 msec per word.

One difficulty with this interpretation is that for Group 2L, the positive and negative functions cross, so that positive RTs are actually shorter than negative ones for the two smallest memory loads (see Figure 1). We shall defer discussion of this problem until the General Discussion. Assuming for the moment that our two-scan interpretation is correct, then it should be possible to eliminate the second scan by providing some a priori basis for distinguishing the two lists. Experiment 2 was designed to test this.

\section{EXPERIMENT 2}

In this experiment, subjects in the experimental condition memorized lists that were distinguished by category: List A always consisted of words representing animate objects, and List B always consisted of words representing inanimate objects. Once a subject decides a probe is positive under this condition, it should no longer be necessary to consult the memorized lists again in order to decide which list the probe belongs to. Consequently, we should expect the slope of the RT function for positive decisions to be reduced to a value very close to that for negative decisions. This condition was compared to a control condition in which the words in the two categories were assigned randomly to the two lists. In this case, we expected the results to resemble those of Condition $2 \mathrm{~L}$ in Experiment 1, with the slope for positive responses being considerably steeper than that for negative responses.

Previous experiments on memory scanning of categorized lists have required binary decisions only, as in the usual Sternberg paradigm. These experiments have shown that having the items in the memorized lists blocked by category reduces the slope of the function relating $\mathrm{RT}$ to total list length (e.g., Naus, 1974; Naus, Glucksberg, \& Ornstein, 1972; Williams, 1971). Naus et al. (1972) proposed that this is because scanning is no longer exhaustive; the subject selects a subset of categories for scanning and terminates scanning when the items in the probed category have been scanned exhaustively. Naus (1974) later showed that with practice and instruction, subjects could select only the items in the probed category for scanning, thus dividing the slope of the RT function by the number of categories.

These results raise the possibility of a confounding influence in our experiment. Categorization might well have the effect of reducing the slope of the RT function in the experimental condition relative to that of the control condition. Consequently, a reduction in slope for positive responses might well be due either to the elimination of the second scan or to curtailment of the exhaustive nature of scanning. However, nonexhaustive scanning should apply to negative as well as to positive responses, so that the difference in slope between positive and negative functions should still bear on whether or not positive decisions require an extra scan.

\section{Method}

Subjects. The subjects were 20 right-handed undergraduates, 14 men and 6 women, aged from 17 to 23 years.

Stimuli and Apparatus. The apparatus was the same as in Experiment 1, except that the words to be memorized were displayed on the screen of a Tektronix 4025 visual display unit, and the probe words were displayed on a Tektronix 604A monitor. Each letter was $4 \mathrm{~mm}$ high and a maximum of $2.2 \mathrm{~mm}$ wide

The stimulus words were selected from a pool of 32 common, concrete, three- to five-letter nouns, representing 16 animate objects and 16 inanimate objects. Each appears at least 19 times per million words of text, as estimated by Thorndike and Lorge's (1944) frequency count, and 22 are in their A or AA lists. With 2 marginal exceptions (LICE and ROCK), all words were scored by Paivio et al. (1968) as being at least 6 (out of 7) on their imagery and concreteness scales and above 6 on the meaningfulness scale. Word length, imagery, concreteness, and meaningfulness were balanced as closely as was practicable between the two categories.

Procedure. The subjects were allocated to two groups, with 7 men and 3 women in each. All subjects learned two lists of words under each of 10 conditions, with list lengths assigned as for Group 2L in Experiment 1 (see Table 1). One group, Group M (mixed-list group), received words chosen randomly from the combined pool of 32 words; thus List A and List B words did not differ systematically with respect to category. For the other group, Group C (categorized-list group), List A words were selected randomly from the pool of words representing animate objects, and List B words were selected randomly from the pool representing inanimate objects. The negative probes were selected randomly from the remaining pool of words.

Instructions were essentially the same as in Experiment 1. That is, subjects were told to press Button $A$ if the test probe belonged to List A, Button B if it belonged to List B, and Button C otherwise. However, subjects in Group $C$ were also told that all words in List A represented animate objects (e.g., CAT, BABY) and all words in List B represented inanimate objects (e.g., COIN, TRUCK).

As in Experiment 1, there were four sessions in which the subjects received all 10 conditions, and the first session served as practice. In all other respects, too, the procedure was the same as in Experiment 1.

\section{Results}

Reaction times. The RTs for correct responses were first subjected to an overall analysis of variance. All three independent variables gave rise to significant main effects: for group $[F(1,18)=7.84, M S e=596,531, p<.01]$, for probe type $[F(2,36)=4.06, M S e=34,199$, $p<.05]$, and for condition $[F(9,162)=52.61, M S \mathrm{e}=$ $29,525, p<.0011$. More importantly, there were significant interactions between group and condition $[F(9,162)=8.38, M S e=29,525 p<.01]$, group and probe type $[F(2,36)=4.32, M S e=34,199, p<.05]$, probe type and condition $[F(18,324)=5.68, M S e=$ $9,016, p<.001]$, and group, probe type, and condition $[F(18,324)=2.80, M S e=9,016, p<.001]$. Mean RTs for each group, probe type, and condition are shown in Table 4.

The main effect of response, and its interactions, were due largely to the contrast between positive (A and B com- 
Table 4

Mean RTs of Responses to Each Probe Type for Each Group Under Each Condition in Experiment 2

\begin{tabular}{rlrlllllllll}
\hline & & \multicolumn{10}{c}{ Condition* } \\
\cline { 2 - 12 } Group & Probe & $1 / 1$ & $1 / 2$ & $2 / 1$ & $2 / 2$ & $1 / 3$ & $3 / 1$ & $3 / 3$ & $2 / 4$ & $4 / 2$ & $4 / 4$ \\
\hline \multirow{3}{*}{ M } & A(Pos) & 538 & 657 & 634 & 728 & 650 & 683 & 890 & 774 & 866 & 1082 \\
& B(Pos) & 586 & 648 & 633 & 778 & 722 & 670 & 930 & 842 & 833 & 1063 \\
& C(Neg) & 633 & 683 & 663 & 725 & 729 & 738 & 792 & 817 & 828 & 916 \\
& A(Pos) & 531 & 573 & 580 & 613 & 592 & 621 & 669 & 679 & 687 & 748 \\
C & B(Pos) & 564 & 610 & 589 & 651 & 648 & 615 & 708 & 681 & 698 & 747 \\
& C(Neg) & 611 & 635 & 638 & 667 & 704 & 675 & 713 & 697 & 748 & 778 \\
\hline
\end{tabular}

*Conditions labeled according to assignment of list lengths to Lists $A$ and $\mathbf{B}$, respectively.

bined) and negative $(C)$ responses [for the main effect, $F(1,36)=4.28, M S e=34,199, p<.05$; for the interaction with group, $F(1,36)=8.63, M S \mathrm{e}=34,199$, $p<.001$; for the interaction with condition, $F(9,324)=$ $8.95, M S e=9,016, p<.001$; and for the triple interaction with group and condition, $F(9,324)=4.71, M S \mathrm{~S}$ $=9,016, p<.001]$. The nature of this last interaction can be seen in Figure 2, which shows that the large slope difference between positive and negative responses, apparent again in Group $M$, was all but eliminated in Group C. This is consistent with our prediction that categorization would eliminate, or at least reduce the incidence of, the second scan for positive decisions.

Although RTs to A probes $(690 \mathrm{msec})$ were slightly shorter than those to B probes $(711 \mathrm{msec})$, the difference was not significant $[F(1,36)=3.84, M S e=34,199$, $.05<p<.10$ ]. The interactions of this contrast with group and with group and condition were negligible $(F \mathrm{~s}<1)$. However, the interaction with condition was significant $[F(9,324)=2.41, M S e=6,019, p<.05]$, although not if one adopts the reduced degrees of freedom $(1,36)$ recommended by Winer $(1971)$ for testing repeated measures effects.
Because of the significant interactions involving group, the data for each group were analyzed separately. These analyses revealed considerably higher error terms in Group M, significant in each case [for probe type, $F(18,18)=5.33, p<.001$; for condition, $F(81,81)=$ $5.41, p<.001$; and for the interaction, $F(162,162)=$ $4.10, p<.001]$.

For Group $\mathrm{M}$, there was a significant main effect of condition $[F(9,81)=30.19, M S e=49,849, p<.001]$, but no significant effect of probe type $[F(2,18)<1]$. The interaction between probe type and condition was significant, however $[F(18,162)=4.75, M S e=14,496$, $p<.001]$, and was attributable primarily to the interaction between condition and positive versus negative probes $[F(9,162)=8.08, p<.001]$; the interaction between condition and $A$ versus $B$ probes was not significant $[F(9,162)=1.43]$. This last result again shows that relative RT to A and B probes did not depend importantly on the distribution of list lengths to the A and $B$ lists.

Because of the interaction between condition and positive versus negative probes, the simple main effect of condition was assessed separately for each probe type; the error term and its degrees of freedom were calculated ac-

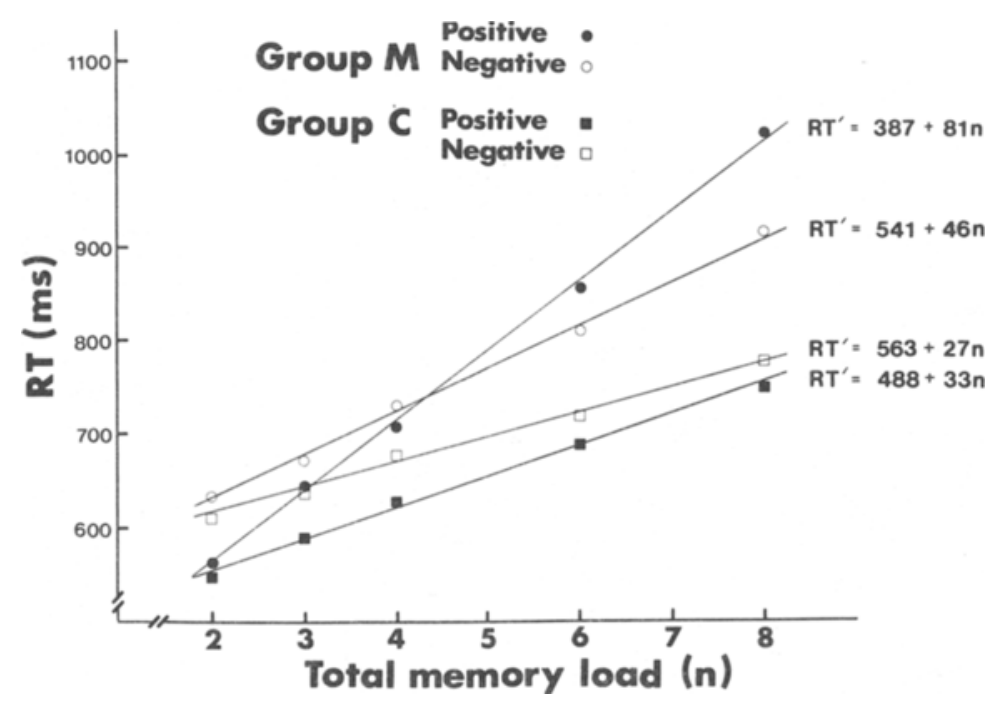

Figure 2. Mean RT to positive and negative probes plotted against memory load, for each group in Experiment 2. 
cording to the formulas given by Winer (1971, pp. 544$545)$ for testing simple main effects. For both positive and negative probes, the simple main effect of condition was significant $[F(9,173)=36.79$ and 11.58 , respectively, $M S e=2,628, p<.001]$.

We again tested the three models defined in Table 1, namely (1) simultaneous scanning of the two lists with constant scan time per word, (2) simultaneous scanning with variable scan time per word, and (3) sequential scanning of the two lists.

For positive probes, these models accounted for $58.80 \%, 80.24 \%$, and $94.29 \%$ of the variance between conditions, respectively. In all three cases, however, the residual was significant $[F(8,173)=17.07,8.14$, and 2.36 , respectively, $p<.05]$. Although the sequentialscanning model provided much the best fit, it also left a small but significant proportion of the variance unexplained. Further analysis showed that this was due primarily to variance between conditions in which the total memory load was the same; specifically, when the total memory load was four words and six words, RTs were significantly longer when the $A$ and $B$ lists were of equal length than when they were unequal $[F(1,173)=5.26$ and 6.85 , respectively, $p<.05$ ]. This effect might be related to differential repetition of the probes, influencing the encoding rather than the scanning stage of processing. It might also be noted that the effect is opposed to the predictions of both simultaneous-scanning models.

For negative probes, the three models accounted for $83.0 \% 95.79 \%$, and $98.10 \%$ of the variance, respectively. The first left a significant residual $[F(8,173)=2.12$, $p<.05]$, but the second two did not (both $F \mathrm{~s}<1$ ). Again, then, the sequential-scanning model provided the best fit. It should be recalled, however, that the second model was somewhat arbitrarily chosen from a population of possible models, and it is conceivable that some other version of a simultaneous-scanning model with variable scan time might provide the best fit.

For Group M, therefore, the results essentially replicate those of Group 2L in Experiment 1 . The slope of the function relating RT to total memory load was $81 \mathrm{msec}$ per word for positive responses and $46 \mathrm{msec}$ per word for negative responses. This difference in slope is again consistent with the notion that positive decisions required a second scan, at an estimated rate of $35 \mathrm{msec}$ per word.

For Group $\mathrm{C}$, there were significant main effects due to probe type $[F(2,18)=23.09, M S e=10,801$, $p<.001]$ and condition $[F(9,81)=32.11, M S e=$ $9,206, p<.001]$. The interaction was significant $[F(18,162)=2.12, M S e=3,534, p<.05]$, unless one adopts the conservative degrees of freedom recommended by Winer (1971). When the effect of probe type was broken down into orthogonal contrasts, RT to List A probes (animate) proved significantly shorter than RT to List B probes (inanimate) $[F(1,18)=6.52, p<.05]$, perhaps because animate words are somehow more salient than inanimate ones. The RT to negative probes was also significantly longer than combined RT to positive probes $[F(1,18)=39.67, p<.001]$.

Although the interaction between probe type and condition was only marginally significant, it was nonetheless analyzed further. The comparison between List $A$ and List B probes did not interact significantly with condition $[F(9,162)=1.89]$, whereas the comparison between positive and negative probes did $[F(9,162)=2.36, p<.05]$, unless one adopts conservative degrees of freedom. Next, the simple main effect of condition was assessed separately for each of positive and negative probes; again, the error term and its degrees of freedom were computed according to the formulas given by Winer (1971, pp. 544-545).

For positive probes, condition was highly significant $[F(9,195)=29.26, M S e=5,424, p<.001]$. The scanning models defined in Table 1 accounted for $77.62 \%$, $93.12 \%$, and $99.04 \%$ of the variance, respectively; the first two left significant residuals $[F(8,195)=10.05$ and 2.22 , respectively, $p<.05]$, but the third did not $[F(8,195)=0.31]$. The sequential-scanning model therefore provided the best fit and yielded an estimated scanning rate of $33 \mathrm{msec}$ per word.

For negative probes, the simple main effect of condition was again highly significant $[F(9,195)=21.71$, $p<.001]$ and the three models accounted for $80.19 \%$, $90.36 \%$, and $88.87 \%$ of the variance, respectively. All three left small but significant residuals $[F(8,195)=4.95$, 2.41 , and 2.78 , respectively, $p<.05]$. In the case of the third (sequential-scanning) model, the residual was again due to significant variation between conditions in which the total memory load was constant $[F(5,195)=3.47$, $p<.05$ ], but further exploration of this failed to reveal any consistent pattern. The estimated scanning rate, assuming sequential scanning, was $27 \mathrm{msec}$ per word.

This slope of $27 \mathrm{msec}$ per word was markedly less than the slope of $46 \mathrm{msec}$ per word for negative responses in Group M. We tested this difference by computing the simple interaction between groups and the linear component of memory load for negative responses only. This interaction was significant $[F(1,157)=11.13, M S e=15,852$, $p<.001]$; the error term and its degrees of freedom were calculated according to the procedures given by Winer (1971). The reduced slope for Group C might be attributed to a restriction on the exhaustiveness of scanning; that is, subjects may have scanned only those words whose category matched that of the probe on some proportion of trials (Naus, 1974). If this were so, however, one would expect RTs for positive probes to depend to some extent on the length of the list corresponding to the probe category; that is, one would expect an interaction between response (A vs. B) and condition. As we have seen, this interaction was not significant $[F(9,162)=1.89, M S e=$ $3,534, .05<p<.10$ l, but it was more nearly so than in Group $\mathrm{M}$ or in Group 2L of Experiment 1. Therefore, we tested the specific hypothesis that the RTs for Group C would be proportional to the list lengths associated with the $A$ and $B$ responses. To do this, we constructed a two- 
dimensional contrast from the lengths of Lists $\mathrm{A}$ and $\mathrm{B}$, centering by rows and columns, and this component of the interaction between response and condition did prove significant $[F(1,162)=6.70, p<.05]$.

Accuracy. Correct responses to each type of probe (A, $B$, or C) were counted for each subject in each group under each condition. These scores were first subjected to an overall analysis of variance. There were significant main effects of probe type $[F(2,36)=21.02, M S e=$ $0.7702, p<.001]$ and condition $[F(9,162)=12.99, \mathrm{MSe}$ $=0.6895, p<.001]$ and significant interactions between group and probe type $[F(2,36)=9.59, M S e=0.7702$, $p<.001]$ and between probe type and condition $[F(18,324)=3.62, M S e=0.4310, p<.05]$. The triple interaction between group, probe type, and condition was also marginally significant $[F(18,324)=1.67, M S e$ $=0.4310, p<.01]$. Percent correct for each group, probe type, and condition are shown in Table 5.

These results again effectively mirror the RT trends; that is, increases in RT are generally associated with decreases in accuracy. This means that the RT trends cannot be attributed to speed-accuracy trade-off.

\section{Discussion}

The results for Group M essentially repeat those for Group 2L in Experiment 1. The functions relating RT to total memory load were again strikingly linear, and the slope was $35 \mathrm{msec}$ per word higher for positive than for negative probes. This agrees closely with the difference of $33 \mathrm{msec}$ per word recorded in Experiment 1, and suggests again that positive decisions require an additional serial exhaustive scan. Again, however, it should be noted that the positive and negative functions cross, so that RTs to positive probes were actually shorter than RTs to negative probes at the smaller memory loads.

For Group $\mathrm{C}$, the difference in slope between positive and negative functions was reduced to a mere $6 \mathrm{msec}$ per word. Thus, when a conceptual basis for distinguishing between $A$ and $B$ responses was supplied, the decision between $A$ and $B$ responses was no longer so dependent upon memory load. In most cases, presumably, subjects were able to make this decision by determining the category of the word, without resorting to scanning or to any other process influenced by the number of words in memory.

It is of interest that the slopes of the negative functions also differed between groups. The slope was $46 \mathrm{msec}$ per word for Group M and 27 msec per word for Group Cless even than the slope of $39 \mathrm{msec}$ per word recorded for Group 1L in Experiment 1. The most likely explanation is that categorization of the lists allowed subjects to restrict scanning on some proportion of trials only to that list representing the category of the probes.

\section{GENERAL DISCUSSION}

In general, the data supported the hypothesis that the subjects who learned two lists scanned them sequentially rather than simultaneously in reaching their decisions (cf. Burrows \& Solomon, 1975; Ryan, 1983). In Group 2L of Experiment 1 and Group $M$ of Experiment 2, relative RT to $A$ and $B$ probes did not vary significantly with the list lengths associated with A and B lists, respectively, suggesting that the subjects did not simply scan the list that contained the probe. In these groups, moreover, RT did not depend simply on the longer of the two lists in each pair, which rules out the hypothesis of simultaneous scanning of the lists with constant scan time per word. The hypothesis of simultaneous scanning with variable scan time is more difficult to test; however, our attempt to simulate an "extreme" version of this hypothesis failed to provide particularly good fits to the data. The best fit was provided by the hypothesis that the subjects scanned sequentially through both lists before reaching their decisions; thus RT was a linear function of total memory load. Given the further assumption that the subjects scanned the lists twice before making positive decisions, but only once before making negative ones, the sequentialscanning hypothesis also yielded estimates of scanning rates that are reasonably consistent with those obtained in regular memory-scanning experiments, or in Group 1L of Experiment 1. The hypothesis of simultaneous scanning approximately halves the estimates of scanning rate, which is another point in favor of sequential scanning.

We do not mean to imply that the variation between conditions was explained completely by variations in total memory load. There was some evidence for variations in RT within memory loads, depending particularly on whether the words were distributed evenly or unevenly between lists. However, this variation was trivial compared with that between memory loads. For Group $2 \mathrm{~L}$ in Experiment 1, for instance, the variation in positive RTs between even and uneven assignment within memory loads accounted for only $2.3 \%$ of the total variation be-

Table 5

Percentage of Correct Responses to Positive and Negative Probes Under Each Condition for Each Group in Experiment 2

\begin{tabular}{clccccccccccc}
\hline & & \multicolumn{10}{c}{ Condition* } \\
Group & Probe & $1 / 1$ & $1 / 2$ & $2 / 1$ & $2 / 2$ & $1 / 3$ & $3 / 1$ & $3 / 3$ & $2 / 4$ & $4 / 2$ & $4 / 4$ \\
\hline \multirow{2}{*}{ M } & Pos & 98.75 & 97.36 & 98.33 & 96.53 & 97.36 & 98.61 & 93.47 & 93.83 & 93.33 & 88.33 \\
& Neg & 98.83 & 99.72 & 99.44 & 99.17 & 99.72 & 99.44 & 98.88 & 98.88 & 99.17 & 99.44 \\
& Cos & 94.44 & 97.92 & 98.19 & 96.52 & 97.50 & 98.19 & 95.56 & 97.13 & 96.11 & 92.78 \\
C & Neg & 99.17 & 98.89 & 98.61 & 99.44 & 96.39 & 98.89 & 98.33 & 97.22 & 96.94 & 95.83 \\
\hline
\end{tabular}


tween conditions, whereas the variation between memory loads accounted for $96.9 \%$. For Group $M$ in Experiment 2 , the corresponding values were $3.7 \%$ and $94.3 \%$, respectively. Of course, these comparisons are biased by the fact that there was greater variation in the dependent variable between memory loads than within them, and to gain further insights into the nature of within-load variations, we plan further experiments with more extreme allocations of list lengths within loads.

For the present, however, we shall focus on the between-load variations, for two reasons: First, only in isolated cases were the within-load variations statistically significant, and we cannot therefore be sure of their true nature or reliability. Second, the between-load effects on RT were strikingly linear, suggesting that their explanation is distinct from that of within-load effects. That is, it seems most reasonable to suppose that between-load effects are due to the scanning stage of processing, whereas within-load effects are more likely due to differential effects of probe repetition, which influence the encoding rather than the scanning stage of processing.

An unanticipated aspect of the results was that, for Group 2L in Experiment 1 and Group $M$ in Experiment 2, the slope for positive responses was almost double that for negative ones, a finding virtually unprecedented in research on memory scanning, as far as we know. Our suggested interpretation of this is that, faced with the choice of three responses, the subjects "parsed" the problem into one of two binary choices. First, they scanned the lists to determine whether the probe was positive or negative, and if it was negative they pressed Button C. If it was positive, then they scanned the lists again in order to determine to which list it belonged and pressed Button A or B, accordingly. In order to test this interpretation, we provided subjects in Group $C$ of Experiment 2 with an a priori basis for distinguishing the lists-words in List A represented animate objects and words in List B represented inanimate objects-and this reduced the difference in slope between positive and negative RT functions to a mere $6 \mathrm{msec}$ per word. Effectively, then, categorization eliminated the second scan, as one would expect if the purpose of the scan is to distinguish which list a positive probe belongs to.

There are, however, some difficulties with our interpretation. One is that the functions for positive and negative probes cross, in both Group $2 \mathrm{~L}$ and Group $\mathrm{M}$; that is, at the smaller list lengths, RTs to positive probes are actually shorter than RTs to negative probes. This is not easy to explain if positive decisions are supposed to require an extra scan. The difference cannot be attributed simply to the physical layout or characteristics of the response buttons; a small pilot study revealed no significant difference in disjunctive RT to the three buttons, and in fact, RT for Button C was intermediate between RT for Buttons A and B. It is possible that the extra time to press Button C occurred at the "response initiation" stage
(Sternberg, 1969b). The subjects might be aware that they are generally slower in reaching positive than negative decisions and compensate by developing a more active response set for $\mathrm{A}$ and $\mathrm{B}$ than for $\mathrm{C}$.

Another problem with the two-scan theory is that the estimated rate of the first scan was higher than that of the second scan and higher than the rates typically obtained in memory-scanning experiments. Some experimenters have found that scanning rate depends on the size of the response set and, in fact, increases as the number of possible responses increases from one to two (Corballis, Roldan, \& Zbrodoff, 1974; Egeth, Marcus, \& Bevan, 1972). A further increase in response set from two to three might produce slower scanning still. This increase would apply to the first postulated scan but not the second, since, according to our interpretation, the number of response alternatives would have been reduced from three to two (A or B) by the time the subject began the second scan.

It might be argued, however, that the first scan is involved in only two decision choices (positive or negative), and that it is the number of possible decisions rather than the number of possible responses that should matter. However, there still remains the decision as to whether or not to make the second scan; the extra load that is present at the time of the first scan may not be one of response set, per se, but may rather be one of maintaining the necessary control structures to see the task through. It might be noted that the estimated rate of the first scan for Group C in Experiment 2 was only $27 \mathrm{msec}$ per word, which, if anything, is less than that normally obtained in memory scanning, even though the number of response alternatives was three in this condition. This lends some support to our interpretation, since we have supposed that the second scan is not necessary under this condition. However, this low scanning rate may also have been due in part to a curtailment of the exhaustiveness of the scan attributable to categorization of the lists (Naus et al., 1972).

If our two-scan interpretation is correct, it is reasonable to ask why the second scan is necessary; a single scan should be sufficient to determine both whether each memorized item matches the probe and whether the match is an A match or a B match. The answer may have to do with capacity limitations. In their work on the verification of sentences describing pictures, Carpenter and Just (1975) proposed a model in which subjects make serial comparisons between constituents of the representation of the picture and corresponding constituents of the representation of the sentence. Whenever a mismatch is found, an index is changed and the serial process begins all over again. To go back to the beginning each time a mismatch is found seems counterintuitive and inefficient, yet the RT data suggest that this is what happens. Carpenter and Just attribute this "reinitialization" to a limitation of short-term memory, implying that the serial comparison process is controlled rather than automatic. 
Similarly, the second serial scan in the two-list conditions in our experiments might be interpreted as a reinitialization due to capacity limitations.

We concede, however, that our interpretation of the data remains speculative, and that other interpretations are possible. In other experiments involving relatively complex discriminations, linear functions relating RT to the length of memorized lists have been obtained with slopes steeper than that derived from the simpler Sternberg paradigm; these include experiments on judgments of recency (Hacker, 1980; Muter, 1979), experiments on discrimination of serial order (Sternberg, 1967), and forced-choice recognition experiments (Hockley, 1984). We might, therefore, suppose that subjects normally make a single, relatively slow scan through the two lists, which explains why the slope for negative probes in Groups $2 \mathrm{~L}$ and $\mathrm{M}$ were steeper than that for Group 1L. On some proportion of positive trials, however, the outcome of the first scan might be ambiguous with respect to list membership, and subjects are forced to make a second scan. Murdock and Anderson (1975) have also suggested that subjects may rescan a list when the outcome of a first scan is ambiguous. The probability of a second scan might increase with memory load, so that there would be relatively few second scans for small memory loads. This would help further to ease the burden of explaining why the positive and negative functions cross, with RTs actually shorter for positive than for negative probes at the smallest loads.

Finally, we note that our data need not be interpreted in terms of serial scanning at all. Although evidence from experiments on the Sternberg paradigm, and variants of it, continue to confirm the linear relation between RT and list length, at least over limited ranges of list lengths, some authors have argued strongly against interpretation in terms of scanning, or have at least shown that there are severe restrictions on the kinds of scanning process that can be plausibly entertained (e.g., Hockley \& Corballis, 1982). For example, the shape of the RT distributions makes it unlikely that scanning is more than an intermittent strategy. Although they do not make the strong prediction that the function relating RT to set-size is linear, theories based on direct access (e.g., Corballis, 1967) or on parallel search (e.g., Ratcliff, 1978) provide adequate accounts of the data; Ratcliff's theory, in particular, provides a good fit to the RT distributions. With respect to the present experiments, a more neutral interpretation of the results is simply that the function relating RT to list length reflects some load-dependent process, not necessarily involving scanning. We may still conclude, however, that in the two-list conditions, except where the lists were distinguished by category, this process was invoked twice, perhaps once to determine whether the probe was positive or negative, and if it was positive, a second time to determine which list it was in.

\section{REFERENCES}

BURRows, D. (1972). Modality effects in retrieval of information from short-term memory. Perception \& Psychophysics, 11, 365-372
Burrows, D., \& OKaDA, R. (1973). Parallel scanning of semantic and formal information. Journal of Experimental Psychology, 97, 254-257.

BurRows, D., \& OKADA, R. (1974). Scanning temporally structured lists: Evidence for dual retrieval processes. Memory \& Cognition, 2, 441-446.

Burrows, D., \&olomon, B. A. (1975). Parallel scanning of visual and auditory information. Memory \& Cognition, 3, 416-420.

CARPEnTer, P. A., \&ust, M. A. (1975). Sentence comprehension: A psycholinguistic processing model of verification. Psychological Review, 82, 45-73.

CoRBALLIS, M. C. (1967). Serial order in recognition and recall. Journal of Experimental Psychology, 74, 99-105.

Corbaluis, M. C. (1975). Access to memory: Analysis of recognition times. In P. M. A. Rabbitt \& S. Dornic (Eds.), Attention and performance (Vol. 5, pp. 591-612) New York: Academic Press.

Corballis, M. C. (1986). Memory scanning: Can subjects scan two lists at once? Psychological Review, 93, 113-114

Corballis, M. C , Katz, J., \& Schwartz, M. (1980). Retrieval from memory sets that exceed the memory span. Canadian Journal of Psychology, 34, 40-48.

Corballis, M. C., Kirby, J., \& Miller, A. (1972). Access to elements of a memorized list. Journal of Experimental Psychology, 94, 185-190.

Corballis, M. C., \& Miller, A. (1973). Scanning and decision processes in recognition memory. Journal of Experimental Psychology, 98, 379-386.

Corballis, M. C , Roldan, C. E., \& Zbrodoff, J. (1974). Response set effects in recognition memory. Memory \& Cognition, 2, 501-508.

DunCaN, J (1980) The locus of interference in the perception of simultaneous stimuli. Psychological Review, 87, 272-300.

EgEth, H., Marcus, N., \& BeVAn, W. (1972). Target-set and responseset interaction: Implications for models of human information processing. Science, 176, 1447-1448.

FoRrIN, B., \& MORIN, R E. (1969). Recognition times for items in short- and long-term memory. Acta Psychologica, 30, 126-141.

HACKER, M. J (1980). Speed and accuracy of recency judgments for events in short-term memory. Journal of Experimental Psychology: Human Learning \& Memory, 6, 651-675.

HOCKLEY, W. E. (1984). Analysis of response time distributions in the study of cognitive processes. Journal of Experimental Psychology: Human Learning \& Memory, 10, 598-615.

Hockley, W. E., Corballis, M. C. (1982). Tests of serial scanning in item recognition. Canadian Journal of Psychology, 36, 189-212.

MURDOCK, B. B., JR., \& ANDERson, R. E. (1975). Encoding, storage, and retrieval of information. In R. L. Solso (Ed.), Information processing and cognition: The Loyola Symposium (pp. 145-194). Hillsdale, NJ: Erlbaum.

MUTER, P. (1979). Response latencies in discriminations of recency. Journal of Experimental Psychology: Human Learning \& Memory $5,160-169$.

NaUS, M. J. (1974). Memory search of categorized lists: A consideration of alternative self-terminating search strategies. Journal of $E x$ perimental Psychology, 102, 992-1000.

Naus, M. J., Glucksberg, S., \& ORnstein, P. A. (1972). Taxonomic word categories and memory search. Cognitive Psychology, 3, 643-654

Norman, D. A., \& Wickelgren, W. A. (1969). Strength theory of decision rules and latency in retrieval from short-term memory. Journal of Mathematical Psychology, 6, 192-208.

Ostry, D., Moray, N., \& Marks, G. (1976). Attention, practice, and semantic targets. Journal of Experimental Psychology: Human Perception \& Performance, 2, 326-336.

Paivio, A., Yuille, J. C , Madigan, S. A. (1968). Concreteness, imagery, and meaningfulness values for 925 nouns. Journal of Experimental Psychology Monographs, 76(1, Pt. 2).

Ratcliff, R (1978). A theory of memory retrieval. Psychological Review, 85, 59-108

RYAN, C. (1983). Reassessing the automaticity-control distinction: Item recognition as a paradigm case. Psychological Review, 90, 171-178.

SCHNEIDER, W., \& SHIFFrIN, R. M. (1977). Controlled and automatic 
processing: I. Detection, search, and attention. Psychological Review, 84, 1-66.

ShIfFrin, R. M., \& SCHNeIder, W. (1984). Automatic and controlled processing revisited. Psychological Review, 91, 269-276.

Sorkin, R. D., Pohlmann, L. E., \& Woods, D. D. (1976). Decision interaction between auditory channels. Perception \& Psychophysics, 19, 290-295.

STERNBERG, S. (1966). High-speed scanning in human memory. Science, 153, 652-654.

STERNBERG, S. (1967). Retrieval of contextual information from memory. Psychonomic Science, 8, 55-56.

STERNBERG, S. (1969a). The discovery of processing stages: Extensions of Donders' method. In W. G. Koster (Ed.), Attention and performance (Vol. 2, pp. 276-315). Amsterdam: North-Holland.

STERNBERG, S. (1969b). Memory-scanning: Mental processes revealed by reaction-time experiments. American Scientist, 57, 421-457.
STERNBERG, S. (1975). Memory scanning: New findings and current controversies. Quarterly Journal of Experimental Psychology, 27, 1-32.

THORNDIKE, E. L., \& LORGE, I. (1944). Teachers' word book of 30,000 words. New York: Columbia University, Teachers' College, Bureau of Publications.

WiLLIAMS, J. D. (1971). Memory ensemble selection in human information processing. Joumal of Experimental Psychology, 88, 231-238.

WINER, B. J. (1971). Principles of experimental design. New York: McGraw-Hill.

(Manuscript received May 21, 1985; revision accepted for publication October 21, 1985.) 